\title{
A case of cystic adenomyosis presenting as uterine malignancy on gross appearance
}

\author{
Dr. M Agarwal, Dr. Puja Kumari \\ Department of Obstetrician \& Gynecology, NEIGRIHMS Shillong, India
}

\begin{abstract}
Adenomyosis of uterus is a relatively à common condition and is usually divided into two main types, the diffuse adenomyosis and focal adenomyosis. However, there is third rare variant called as cystic adenomyosis which is due to repeated focal haemorrhages and results in cystic spaces filled with altered blood. Here, we are presenting a unique case of 25 years old girl who presented with severe dysmenorrhoea with 26 weeks abdominal lump. USG showed uterine fibroid with right ovarian chocolate cyst. Patient was planned for myomectomy with cystectomy. Intraoperatively large, irregular solid cystic mass $(25 \times 15 \times 10 \mathrm{~cm} 3)$ was removed which appeared malignant and total hysterectomy with bilateral salpingectomy was done. But mass was identified as cystic adenomyosis associated with diffuse adenomyosis of uterus on histopathology. Thus, although rare, but adenomyosis may be abnormally enlarged, solid, irregular, malignant in appearance and MRI may be required preoperatively for correct diagnosis and plan of management.
\end{abstract}

Keywords - Cystic adenomyosis, diffuse adenomyosis, Focal adenomyosis, Juvenile adenomyosis, Solid cystic mass.

\section{INTRODUCTION}

Adenomyosis of uterus is a relatively common condition and is usually divided into two main types, the diffuse adenomyosis and focal adenomyosis. However there is third rare variant called as cystic adenomyosis which is due to repeated focal haemorrhages and results in cystic spaces filled with altered blood .Here we are presenting a case of 25 years old girl who presented with severe dysmenorrhoea with 26 weeks abdominal lump, which appeared as uterine malignancy per operatively but was identified as cystic adenomyosis on histopathology.

\section{CASE REPORT}

A 25 year old unmarried lady presented with c/o heavy menstrual bleeding since 1 year with pain in lower abdomen for 6 months. On examination, patient was severely anaemic (HB 5.5g \%) with large uterine mass of 24 weeks size which was firm, mobile and tender. USG showed uterine fibroid of size $(9.6 \mathrm{~cm} \times 9.8 \mathrm{~cm})$

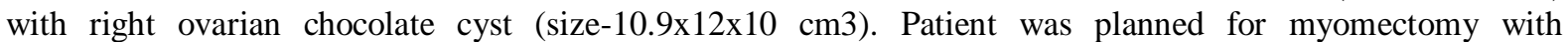
cystectomy. But intraoperatively large irregular solid cystic mass of $25 \times 15 \times 10 \mathrm{~cm} 3$ arising from fundus of uterus and extending into upper abdomen and adhered to omentum was seen; which grossly appeared malignant. Right ovarian chocolate cyst of 10x10 $\mathrm{cm} 2$ size was noticed. Surgery was proceeding with total abdominal hysterectomy with bilateral salpingectomy. HPE report showed uterine size of $16 \times 14 \times 11 \mathrm{~cm} 3$ with growth arising from fundus $(25 \times 13 \times 10 \mathrm{~cm} 3)$ grossly. On cut section, the mass was showing multiple solid cystic areas and cyst was filled with hemorrhagic fluid. Microscopic features were suggestive of cystic adenomyosis. Patient recovered well postoperatively and was discharge on tenth day post op. She came for follow up after 1 month and was symptom free.

\section{RESULTS AND DISCUSSIONS}

Cystic adenomyosis is a rare cause of severe dysmenorrhoea. Kriplani A et al have stated that all patients, reported in the literature and in their series, were younger than 30 years which they suggest, can be considered as arbitrary cut off point to differentiate juvenile from adult cystic adenomyosis ${ }^{1}$. Our patient was also 25 years old, so she can be considered as case of juvenile cystic adenomyosis. Most of these patients present with severe dysmenorrhoea with or without huge abdominal mass. Imaging modalities as ultrasonography, CT scan and MRI may not be able to identify the condition correctly.

In a case report by Kamio M. et al TVS and MRI showed a $3 \times 3 \mathrm{~cm} 2$ cystic mass within left anterior wall of uterine corpus. The case was preliminarily thought to be cavitated rudimentary uterine horn. But after operating, they found a hemorrhagic adenomyosis cyst measuring $3 \mathrm{~cm}$ within the left anterior wall of uterine corpus. $^{2}$

Ho ML et al also reported a similar case where ultrasonography demonstrated a large cystic lesion, which was localised to the myometrium on CT scan and MRI; the lesion was diagnosed to be cystic adenomyosis by pathology after surgical resection. 
In our case also, ultrasonography of the abdominal lump suggested it to be fibroid with endometriosis. Per operatively the gross specimen appeared as though it is a case of malignancy (as seen in picture).

Diagnosis was established after histopathology. Although it had appeared to be grossly malignant case, but finally turned out to be adenomyosis. Had it been locally confined cystic adenomyosis, a resection would have been sufficient. But in this case, as cystic mass was accompanied with diffuse adenomyosis of uterus, hysterectomy was required.

English DP et al have reported a case where USG revealed cystic structure, homogenously echogenic in the anterior corpus of uterus measuring $2.7 \times 2.4 \times 3.5 \mathrm{~cm}$. The patient's symptoms improved after transvaginal aspiration. According to them, it was an adenomyosis cyst in clinical presentation, and location was within myometrium with gross appearance of chocolate cyst. ${ }^{4}$

Figure: Cut Section showing Adenomyosis cyst with Uterus

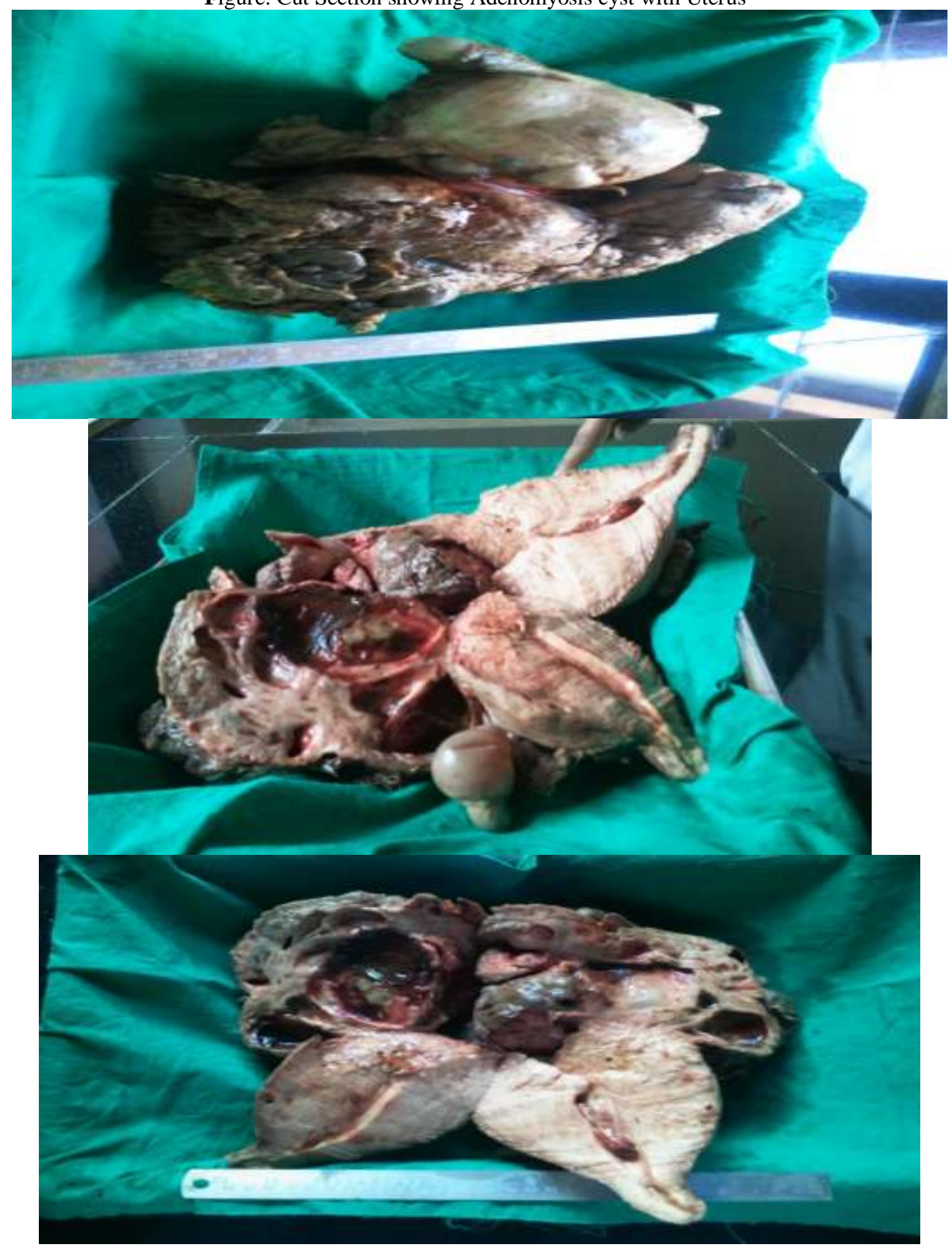




\section{CONCLUSION}

Awareness of this condition is important for timely and accurate diagnosis so that it can be followed by appropriate intervention. Although very uncommon but adenomyosis may be abnormally enlarged, solid and malignant in appearance. Ultrasound may not be accurate in diagnosis. So, MRI may be required for correct diagnosis and for further plan of action. As Kataoka $M L$ et al has described features of three cases of adenomyosis cysts on MRI. Two cases were subserosal and one was intramyometrial. All three cases had cystic spaces filled with hyper intense fluid on T1-weighted images, which were surrounded by hypo intense tissue on T2-weighted images. ${ }^{5}$

Intervention required may be in form of ultrasound guided aspiration of cyst in the uterus or hysterectomy depending on the severity of symptoms.

\section{Acknowledgements}

The author would like to acknowledge Dr A Santa Singh, Head of Department of Obstetrics and Gynecology, for his guidance and support.

\section{Journal Papers:}

\section{REFERENCES}

[1] Kriplani A, Mahey R, Agarwal N, Bhatla N, Yadav R, Singh MK. Laparoscopic management of juvenile cystic adenomyoma: four cases. J Minim Invasive Gynecol. 2011 May-Jun;18(3):343-8.

[2] Kamio M, Taguchi S, Oki T, Tsuji T, Iwamoto I, Yoshinaga M, Douchi T. - J Obstet Gynaecol Res. Isolated adenomyotic cyst associated with severe dysmenorrhea 2007 Jun;33(3):388-91.

[3] Ho ML, Ratts V, Merritt D. Adenomyotic cyst in an adolescent girl. Journal of Pediatric and Adolescent Gynecology 22(3): e33- e38, 2009.

[4] English DP, Verma U, Pearson JM.Uterine cyst as a cause of chronic pelvic pain: a case report. J Reprod Med. 2012 Sep-Oct;57(9$10): 446-8$.

[5] Kataoka ML, Togashi K, Konishi I, Hatabu H, Morikawa K, Kojima N, et al. MRI of adenomyotic cyst of the uterus. J Comput Assist Tomogr 1998; 22:555-9. [PubMed] J Comput Assist Tomogr. 1998 Jul-Aug; 22(4):555-9. 